\title{
Estudo numérico de um arranjo triangular de cilindros submetidos a escoamento laminar com convecção forçada
}

\author{
Numerical Study of an triangular arrangement of cylinders submitted to forced convective \\ laminar flow
}

\author{
S. G. da Fonseca ${ }^{1}$; F. R. Cardoso ${ }^{3}$; A. L. Razera ${ }^{3}$; L. A. Isoldi ${ }^{3}$; A. F. Porte ${ }^{2}$; L. \\ A. O. Rocha ${ }^{4}$; E. D. dos Santos ${ }^{3}$; M. M. Galarça, ${ }^{2, *}$ \\ ${ }^{\text {I}}$ Programa de Pós-Graduação em Modelagem Computacional, Universidade Federal do Rio Grande (FURG), \\ 96201-900, Rio Grande - RS, Brasil \\ ${ }^{2}$ Instituto Federal de Educação, Ciência e Tecnologia do Rio Grande do Sul, 96201-460, Rio Grande - RS, Brasil \\ ${ }^{3}$ Escola de Engenharia, Universidade Federal do Rio Grande (FURG), 96201-900, Rio Grande - RS, Brasil \\ ${ }^{4}$ Departamento de Engenharia Mecânica, Universidade Federal do Rio Grande do Sul (UFRGS), 90050-170, Porto \\ Alegre-RS, Brasil
}

*marcelo.galarca@ riogrande.ifrs.edu.br

(Recebido em 22 de setembro de 2014; aceito em 29 de dezembro de 2014)

\begin{abstract}
O presente trabalho estuda a transferência de calor por convecção forçada em um arranjo triangular de cilindros considerando um escoamento em regime laminar, bidimensional, incompressível e transiente. A solução numérica foi obtida a partir de um código computacional baseado no método dos volumes finitos (MVF). Para a otimização geométrica do arranjo de cilindros é empregado o Método Constructal Design. As simulações foram realizadas para escoamentos com números de Reynolds e de Prandtl de $\operatorname{Re}_{D}=100 \mathrm{e}$ $\operatorname{Pr}=0.71$, respectivamente. A otimização parte da avaliação de qual razão $S_{T} / D$ (passo transversal sobre o diâmetro) e $S_{L} / D$ (passo longitudinal sobre o diâmetro) que minimiza o coeficiente de arrasto e maximiza a taxa de transferência de calor (número de Nusselt) entre os cilindros e o escoamento circundante. Os resultados mostraram que o comportamento fluidodinâmico e o térmico foram bastante influenciados pelas razões $S_{T} / D$ e $S_{L} / D$. O coeficiente de arrasto $\left(C_{D}\right)$ mínimo foi encontrado para a configuração $S_{L} / D=$ 5,0 e $S_{T} / D=1,5$, e o máximo número de Nusselt $\left(N u_{D}\right)$ foi obtido para $S_{L} / D=1,5$ e $S_{T} / D=5,5$. Entretanto, os melhores arranjos considerando a função multi-objetivo (arrasto e transferência de calor) foram alcançados em uma região ótima e não para uma configuração específica. Dentre os casos analisados se destacaram as configurações $S_{L} / D=5,0-S_{T} / D=2,0 ;$ e $S_{L} / D=5,0-S_{T} / D=2,5$, sendo que os dois primeiros valores trouxeram benefícios para o problema fluidodinâmico e os dois últimos valores apresentaram uma leve vantagem do ponto de vista térmico.

Palavras-chave: otimização geométrica, coeficiente de arrasto, número de Nusselt, Constructal Design
\end{abstract}

The present work studies the convection heat transfer by forced convection through a triangular arrangement for three cylinders considering a laminar, two dimensional, incompressible and unsteady flow. Regarding to the numerical solution for the main equations, the solution is ran by using a computational code based on the FVM (Finite Volume Method). The Constructal Design method is applied to optimize the geometry arranged for the three cylinders. Simulations were run for flows with Reynolds and Prandtl numbers of 100 and 0.71, respectively. The optimization considers which ratio $S_{T} / D$ (transversal step over diameter) and $S_{L} / D$ (longitudinal step over diameter) minimize the drag coefficient and maximize the heat transfer rate (from Nusselt number) between the cylinders and the surrounding cross flow. Results showed that the fluid dynamics and thermal behavior were heavily influenced by the ratios $S_{T} / D$ and $S_{L} / D$. The minimum drag coefficient $(C d)$ was found for the configuration $S_{L} / D=5.0$ and $S_{T} / D=1.5$, while the maximum Nusselt number $(N u D)$ lies over the $S_{L} / D=1.5$ and $S_{T} / D=5.5$ ratios. However, the best arrangements considering the multi-objective function (drag and heat transfer) were achieved into an optimal region and not for a specific configuration. Among the analyzed cases, can be highlighted the $S_{L} / D=5.0-S_{T} / D=2.0$; and $S_{L} / D=5.0-S_{T} / D=2.5$ configurations. The first two values presented benefits for the fluid dynamic problem and the last ones showed a slight advantage for the thermal process.

Keywords: geometrical optimization, drag coefficient, Nusselt number, Constructal Design 


\section{INTRODUÇÃO}

O atual desenvolvimento dos mais diversos equipamentos utilizados no dia a dia, como geladeiras, fornos, condicionadores de ar, entre outros, remete a importância de pesquisas para atingir uma melhor eficiência destes equipamentos, bem como o enfoque no consumo de energia, que é uma necessidade humana e um bem comum a todos. Foram direcionados muitos esforços para economizar energia por otimização de seu uso, principalmente em procedimentos industriais, onde este sistema é utilizado em quase todos os processos.

Nos problemas de engenharia a área da transferência de calor é considerada de extrema importância. Dentre esses problemas podem-se citar alguns, como: operação e manutenção de trocadores de calor, que são comumente encontrados em centrais térmicas, instalações de aquecimento de água e refrigeração de radiadores de veículos automotores; isolamentos térmicos para minimização da transferência de calor em equipamentos térmicos, câmaras frias e para conforto térmico em ambientes de trabalho e residencial; entre outros [1,2].

Ao longo dos anos vem sendo desenvolvido diversos trabalhos de pesquisas onde o tema escoamento sobre corpos imersos em fluidos é abordado para diversas finalidades, onde é possível citar os escoamentos com transferência de calor, por exemplo, trocadores de calor e reatores nucleares, ou em escoamentos isotérmicos, os quais remetem a problemas de engenharia do vento e projeto de estrutura [3,4].

Em [5] foi realizado um estudo numérico sobre a disposição de dois cilindros com o mesmo diâmetro $D$ em um arranjo em tandem, inseridos em um escoamento com valores de $R e_{D}$ iguais a 100 e 200, laminar e bidimensional, onde foram investigadas várias distâncias entre centros $L$ $(1,5 D \leq L \leq 10 D)$. Os resultados evidenciaram uma relevante mudança na fenomenologia do fluxo para $L$ igual $4 D$, o que ocasionou um aumento significativo nas forças de arrasto e no número de Strouhall. Além disso, quando os vórtices dos dois cilindros estão em fase, à força de sustentação máxima é alcançada.

No trabalho de [6] foi considerado um par de cilindros e um escoamento por convecção natural, onde foi avaliada a geometria que maximiza a taxa de transferência de calor. Neste estudo foi observado que os diâmetros dos cilindros otimizados são insensíveis a mudança do número de Rayleigh (força de trabalho) e na medida em que a estrutura geométrica tornou-se mais complexa a taxa de transferência de calor aumenta.

Outro estudo numérico sobre um par de cilindros, submetidos a um escoamento turbulento, com $R e_{D}$ igual a 22000 e $P r$ igual a 0,71 , bidimensional, no regime permanente, com convecção forçada é apresentado em [7]. Para a avaliação da geometria foi utilizado o método de Constructal Design. Na abordagem da turbulência foi empregado o modelo Simulação de Grandes Escalas (LES - do inglês: Large Eddy Simulation). O principal objetivo foi avaliar qual ângulo entre o par de cilindros $(\beta)$ com a direção da corrente livre do escoamento que maximiza a taxa de transferência de calor e minimiza a força de arrasto através dos cilindros e escoamento circundante. Os resultados apontaram que os campos fluidodinâmicos e térmicos tiveram seu comportamento fenomenológico fortemente influenciado pelo arranjo geométrico do par de cilindros. O coeficiente de arrasto mínimo $\left(C_{D}\right)$ e o coeficiente de transferência de calor máximo $\left(N u_{D}\right)$ são obtidos para valores de $\beta$ iguais a $0^{\circ}$ e $90^{\circ}$, respectivamente. No entanto, para o caso multi-objetivo $\left(\left(C_{D}\right)\right.$ mínimo e $\left(N u_{D}\right)$ máximo $)$ foi obtido para valores do ângulo $45^{\circ} \leq \beta \leq 60^{\circ}$. Já em [8] foi realizado um estudo da interação de um escoamento laminar, bidimensional, com um número de Reynolds $R e_{D}$ igual a 200 em um arranjo de três cilindros, com o objetivo de controlar os esforços na estrutura dos cilindros, conhecido na literatura como controle passivo da esteira. Dois destes cilindros com um diâmetro de $D / 8$, sendo $D$ o diâmetro do cilindro principal, são posicionados simetricamente na esteira de um cilindro principal, induzindo alterações significativas na topologia do escoamento e nos respectivos coeficientes aerodinâmicos. Com a variação da posição dos cilindros de controle na direção do escoamento descobriu-se que o efeito de controle se mantém até a faixa de 2,5 a 2,8 $\mathrm{D}$ do cilindro principal. Observou-se também que os esforços sobre os cilindros e a topologia do escoamento mudam completamente ao atingir uma distância crítica e, para valores inferiores a esta distância, os esforços são expressivamente menores que os valores obtidos para um cilindro isolado. Após a 
distância crítica, os esforços flutuantes atingem valores superiores aos valores encontrados para um cilindro isolado.

Um estudo de otimização geométrica de um arranjo triangular com três cilindros em um escoamento laminar, para um problema multi-objetivo (minimização da força de arrasto e maximização da taxa de transferência de calor) através do método Constructal Design, pode ser encontrado em [7]. Foram simulados os valores para as razões de $S_{L} / D$ iguais a 3,5 (fixo) e $S_{T} / D$ variando entre 1,5 e 5,0. Os resultados mostraram que o comportamento fluidodinâmico e térmico foi bastante influenciado pela razão $S_{\mathrm{T}} / D$. O coeficiente de arrasto $\left(C_{D}\right)$ mínimo e o máximo número de Nusselt $\left(N u_{D}\right)$ são obtidos para $S_{\mathrm{T}} / D$ iguais 1,5 e 5,5 , respectivamente. Contudo, os melhores arranjos considerando a função multiobjetivo (arrasto e transferência de calor) foram alcançados na região $S_{\mathrm{T}} / D$ de aproximadamente 2,0 , mais próximo do ótimo obtido para o problema fluidodinâmico.

Dentro deste contexto e dando continuidade ao estudo realizado em [7], o objetivo principal do presente trabalho é determinar as razões ótimas, variando novas faixas de $S_{L} / D$ (passo longitudinal sobre o diâmetro do cilindro) e $S_{T} / D$ (passo transversal sobre o diâmetro do cilindro), que maximiza o coeficiente de transferência de calor e minimiza o coeficiente de arrasto, ou seja, um problema multi-objetivo, em um arranjo triangular de cilindros inseridos em um escoamento transiente e laminar, cruzado, com transferência de calor por convecção forçada.

\section{MODELAGEM MATEMÁTICA E NUMÉRICA}

A modelagem dos escoamentos incompressíveis, transientes com convecção forçada no regime laminar é baseada na solução das equações de Navier-Sokes (conservação de massa e de quantidade de movimento) de conservação energia, em conjunto com as condições de contorno e iniciais do problema. Estas equações de conservação são apresentadas, respectivamente, como [1]:

$$
\begin{array}{lr}
\frac{\partial v_{j}}{\partial x_{j}}=0 & (j=1 \text { e } 2) \text { em } t \times \Omega \\
\frac{\partial}{\partial t}\left(v_{i}\right)+\frac{\partial}{\partial x_{j}}\left(v_{i} v_{j}\right)+\frac{1}{\rho} \frac{\partial P}{\partial x_{j}} \delta_{i j}-\frac{\partial}{\partial x_{j}}\left\{v\left(\frac{\partial v_{i}}{\partial x_{j}}+\frac{\partial v_{j}}{\partial x_{i}}\right)\right\}=0 & (i, j, k=1 \text { e } 2) \text { em } t \times \Omega \\
\frac{\partial T}{\partial t}+\frac{\partial}{\partial x_{j}}\left(v_{j} T\right)-\frac{\partial}{\partial x_{j}}\left\{\alpha \frac{\partial T}{\partial x_{j}}\right\}-q^{\prime \prime \prime}=0 & (j=1 \text { e } 2) \text { em } t \times \Omega
\end{array}
$$

onde $\rho$ é a massa específica do fluido $\left(\mathrm{kg} / \mathrm{m}^{3}\right) ; \mu$ é a viscosidade dinâmica do fluido $(\mathrm{kg} /(\mathrm{ms})) ; v$ é a viscosidade cinemática do fluido $\left(\mathrm{m}^{2} / \mathrm{s}\right) ; \alpha$ é a difusividade térmica $\left(\mathrm{m}^{2} / \mathrm{s}\right) ; v_{i}$ é a velocidade do fluido na direção $i, i$ variando de 1 a $2(\mathrm{~m} / \mathrm{s}) ; x_{\mathrm{i}}$ corresponde a coordenada espacial na direção $i, i$ variando de 1 a $2(\mathrm{~m}) ; P$ é a pressão $\left(\mathrm{N} / \mathrm{m}^{2}\right) ; T$ é a temperatura $\left({ }^{\circ} \mathrm{C}\right.$ ou $\left.\mathrm{K}\right) ; \delta_{i j}$ é o operador delta de Kronecker; $\Omega$ é o domínio espacial (m); $t$ representa o domínio de tempo (s) e $q^{\prime \prime \prime}$ é o termo fonte de energia, que para os casos simulados no presente trabalho é nulo.

As Equações (1) - (3) são resolvidas através de um software comercial de dinâmica dos fluidos computacional (CFD - do inglês: Computational Fluid Dynamics) que é baseado no método de volumes finitos (FVM - do inglês: Finite Volume Method) [11]. Em todas as simulações foram empregados volumes finitos retangulares. O acoplamento pressão-velocidade foi realizado com o uso do algoritmo SIMPLE e para o tratamento dos termos advectivos foi empregado o esquema de interpolação Upwind de segunda ordem para as equações de conservação de quantidade de movimento e de energia. Além disso, os cálculos foram considerados convergidos quando os resíduos para as equações de conservação de massa, de quantidade de movimento e de energia entre duas iterações consecutivas foram menores do 
que $10^{-6}, 10^{-6}$ e $10^{-8}$, respectivamente. Maiores detalhes relacionados com o FVM podem ser encontrados em $[12,13]$.

As simulações numéricas foram realizadas usando um computador com dois processadores dual-core Intel com $2.67 \mathrm{GHz}$ de clock e 8GB de memória RAM. Para a paralelização foi utilizada a biblioteca de passagem de mensagem (MPI - do inglês: Message Passing Interface). O tempo de processamento para cada simulação foi de aproximadamente $1,16 \times 10^{3} \mathrm{~s}$.

A independência de malha para cada escoamento é alcançada quando o desvio relativo entre o número de Nusselt $\left(N u_{D}\right)$ médio obtido nos três cilindros para duas malhas consecutivas atende ao seguinte critério $\left(\left|N u_{D}{ }^{j+1}-N u_{D}{ }^{j}\right|\right) / N u_{D}{ }^{j}<5 \times 10^{-3}$, onde o superíndice $j$ refere-se a malha grosseira e o superíndice $j+1$ a malha mais refinada. Os resultados do teste de independência de malha, bem como o tempo de processamento $\left(t_{\text {proces }}\right)$ para cada malha simulada são apresentados na Tabela 1 . Em todos os casos estudados foi empregado um passo de tempo de $\Delta t=1 \times 10^{-3} \mathrm{~s}$ e o tempo final de simulação foi $t_{\mathrm{f}}=2,0 \mathrm{~s}$.

Tabela 1: Teste de independência de malha para um escoamento sobre três cilindros a $\operatorname{Re}_{D}=100, \operatorname{Pr}=$

\begin{tabular}{|c|c|c|c|}
\hline \multicolumn{4}{|c|}{$0,71, S_{T} / D=5,5$ e $S_{L} / D=5,0$} \\
\hline Número de Volumes & $N u_{D}$ & $\left(\left|N u_{D}^{j+1}-N u_{D}^{j}\right|\right) / N u_{D}^{j}$ & $t_{\text {proces }}(\mathrm{s})$ \\
\hline 24000 & 5,5121 & $3,71 \times 10^{-2}$ & 4200 \\
\hline 47604 & 5,3073 & $7,36 \times 10^{-3}$ & 5760 \\
\hline 63386 & 5,2683 & $4,65 \times 10^{-3}$ & 11640 \\
\hline 148396 & 5,2438 & -------------- & -------- \\
\hline
\end{tabular}

Com o objetivo de avaliar a metodologia numérica empregada foi simulado um escoamento incompressível, transiente, laminar e com convecção forçada para $R e_{D}$ igual a 100 e Pr igual a 0,71 . O número de Nusselt médio foi comparado com resultados de correlações experimentais apresentadas na literatura $[14,15]$. Os resultados de comparação são apresentados na Tabela 2 e apresentaram um desvio máximo de aproximadamente $1,5 \%$. Estudos preliminares de verificação e validação da metodologia numérica empregada no presente trabalho foram realizados e, por simplicidade, não serão reproduzidos aqui $[7,16]$.

Tabela 2: Validação de metodologia numérica para obtenção do número de Nusselt em um escoamento a

\begin{tabular}{ccc} 
& \multicolumn{2}{c}{$\operatorname{Re}_{D}=100$ e $\operatorname{Pr}=0,71$} \\
\hline Referência & $\mathbf{N u}_{\mathbf{D}}$ & Desvio (\%) \\
\hline Presente trabalho & 5,2683 & ----- \\
Hilpert [14] & 5,2100 & 0,96 \\
Churchill e Bernstein [15] & 5,1800 & 1,54 \\
\hline
\end{tabular}

\section{DESCRIÇÃO DO PROBLEMA}

A Figura 1 apresenta o domínio do escoamento, onde é possível observar as razões $S_{T}$ (passo transversal) e $S_{L}$ (passo longitudinal) que serão avaliadas no presente trabalho. Foi considerado um escoamento sobre um arranjo triangular de cilindros com o mesmo diâmetro $D$ igual a 0,1 m. O domínio computacional possui as seguintes dimensões: $L=25,0 D=2,5 \mathrm{~m} ; H=15,0 D=$ $1,5 \mathrm{~m}$ e $L_{1}=8 D=0,8 \mathrm{~m}$. As superfícies dos cilindros possuem a condição de contorno de nãodeslizamento e impermeabilidade $\left(u_{\mathrm{w}}=0 \mathrm{~m} / \mathrm{s}\right)$. As superfícies, inferior e superior possuem uma condição de simetria e a superfície lateral direita possui uma condição de contorno de tensão nula $(\tau=0)$. Com relação ao comportamento fluidodinâmico do escoamento, o mesmo é gerado pela imposição de um perfil uniforme de velocidades constante $u_{\infty}$ igual a $50 \mathrm{~m} / \mathrm{s}$ (velocidade na corrente livre) na superfície de entrada. Para o problema térmico, o aquecimento do fluido é causado pela diferença de temperatura entre a corrente livre do escoamento $\left(T_{\infty}=20^{\circ} \mathrm{C}\right)$ que é constante, e uniforme, imposta na superfície de entrada, e a temperatura dos cilindros $\left(T_{\mathrm{w}}=30\right.$ $\left.{ }^{\circ} \mathrm{C}\right)$, também uniforme e constante. 


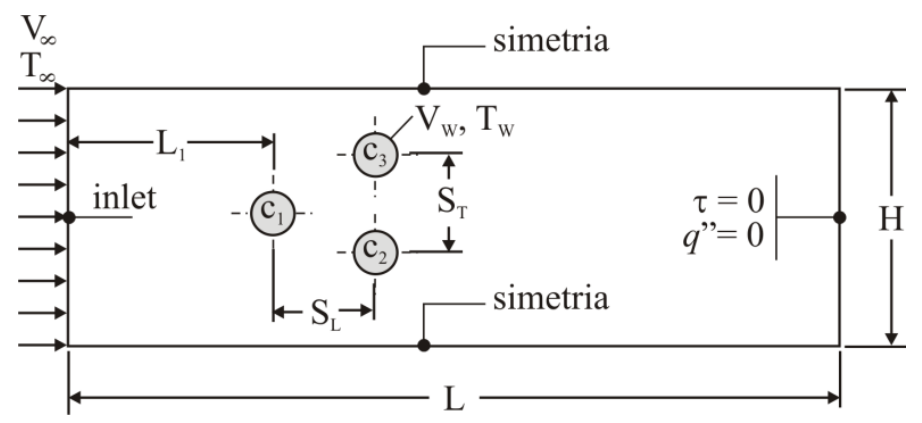

Figura 1: Domínio computacional do escoamento sobre arranjo triangular de cilindros

Para a otimização geométrica do problema é empregado o método Constructal Design $[7,9$, 10]. O Constructal Design é um método baseado no princípio de objetivos e restrições (locais e globais) sendo o método usado para aplicação da Lei Constructal. A Lei Constructal afirma que qualquer sistema de fluxo com dimensões finitas para persistir ao longo do tempo (para sobreviver) deve evoluir suas configurações geométricas de tal forma que facilite o acesso as correntes internas através do sistema.

Para o presente estudo, foram definidas como restrições geométricas do problema: 1) a área ocupada pelos três cilindros, que é a mesma em todos os casos estudados $\left(A_{t}=A_{c 1}+A_{c 2}+A_{c 3}\right)$ os diâmetros dos cilindros são iguais $\left(D_{1}=D_{2}=D_{3}=D\right)$; e 3) o espaço ocupado pelo par de cilindros a jusante do primeiro cilindro não deve ser maior do que o domínio computacional $\left(S_{T}\right.$ $+D<H$ ). O problema possui dois graus de liberdade: $S_{T} / D$ e $S_{L} / D$ e duas funções objetivo: 1 ) a maximização do número de Nusselt $\left(N u_{D}\right)$ sobre o arranjo de cilindros e a minimização do coeficiente de arrasto $\left(C_{D}\right)$.

O processo de otimização é dividido em dois passos, conforme mostrado na Figura 2. No primeiro passo, a geometria é otimizada pela variação do grau de liberdade $S_{\mathrm{T}} / D$ e mantendo-se fixo o parâmetro $S_{\mathrm{L}} / D$. O maior valor de número de Nusselt $\left(N u_{D}\right)$ será a geometria ótima para o problema térmico e o menor valor para o coeficiente de arrasto $\left(C_{D}\right)$ e o parâmetro $S_{\mathrm{T}} / D$ correspondente será o parâmetro uma vez otimizado, $\left(S_{\mathrm{T}} / D\right)_{0}$. No segundo passo, o mesmo processo é repetido para vários valores de $S_{\mathrm{L}} / D$. No presente estudo, o parâmetro $S_{\mathrm{L}} / D$ é variado na faixa $1,5 \leq S_{\mathrm{L}} / D \leq 5,0$, enquanto o parâmetro $S_{\mathrm{T}} / D$ é variado na seguinte faixa $1,5 \leq$ $S_{\mathrm{T}} / D \leq 5,5$. O conjunto de resultados de $S_{\mathrm{L}} / D$ igual a 3,5 foi estudado anteriormente por [7] e compõe o estudo completo aqui apresentado.

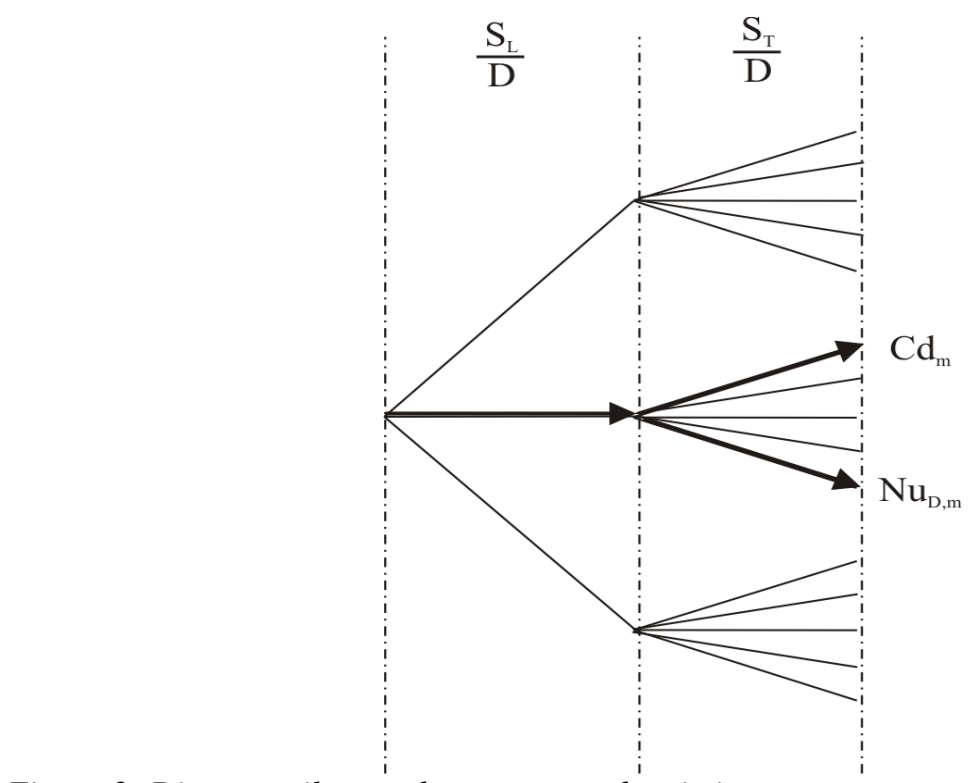

Figura 2: Diagrama ilustrando o processo de otimização para um problema com dois graus de liberdade 


\section{RESULTADOS E DISCUSSÕES}

Conforme citado na seção anterior, foram avaliadas numericamente as influências das razões $S_{\mathrm{L}} / D$ e $S_{\mathrm{T}} / D$ sobre o escoamento térmico e fluidodinâmico do escoamento laminar com $\operatorname{Re}_{\mathrm{D}}$ igual a 100 e Pr igual a 0,71. A Figura 3 mostra, para cada passo longitudinal constante $\left(S_{L} / D\right)$, as variações dos parâmetros $\left(S_{\mathrm{T}} / D\right)$. A otimização do problema multi-objetivo busca a minimização do coeficiente de arrasto $\left(C_{D}\right)$ e a maximização do coeficiente de transferência de calor $\left(N u_{D}\right)$, para o arranjo de cilindros.

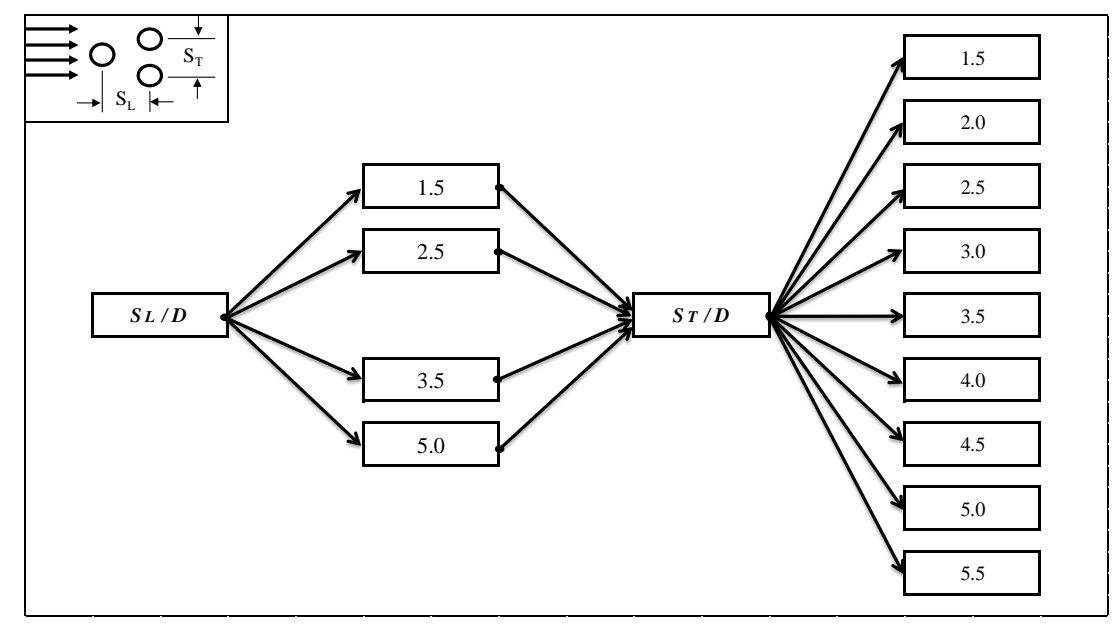

Figura 3: Conjunto de simulações realizadas

Observando os resultados obtidos em [7] onde foram realizados os estudos para a variação do passo transversal $S_{T} / D$ enquanto o passo longitudinal foi mantido constante, $S_{L} / D$ igual a 3,5 . A Figura 4a apresenta o comportamento do coeficiente de arrasto $\left(C_{D}\right)$ analisado em cada cilindro como função das razões $S_{T} / D$. É observado que o arrasto aumenta à medida que o passo transversal também aumenta. Para os cilindros 2 e 3 o $C_{D}$ apresenta comportamento assintótico e crescente com o aumento da razão $S_{T} / D$. O resultado de assimetria obtido para o cilindro $3 \mathrm{em}$ comparação com o cilindro 2 é explicado pela formação dos campos de pressão atrás dos cilindros 2 e 3. Para razões inferiores a $S_{\mathrm{T}} / \mathrm{D}$ de aproximadamente 2,0 é possível observar que o cilindro $1\left(C_{l}\right)$ possui uma resistência muito maior ao escoamento, enquanto os cilindros 2 e 3 ficam "escondidos" atrás do cilindro 1, se localizando em uma região de baixa pressão. Esse comportamento faz com que o coeficiente de arrasto seja muito maior para o cilindro 1 do que para os demais. Conforme a razão $S_{T} / D$ aumenta (com os cilindros mais afastados) os cilindros posteriores $\left(C_{2}\right.$ e $\left.C_{3}\right)$ passam a ser influenciados pela ação direta do escoamento da corrente livre, aumentando o seu coeficiente de arrasto até um ponto onde o comportamento se estabiliza (para $S_{T} / D \geq 4,5$ ).

A relação entre $N u_{D}$ e a razão $S_{T} / D$ é apresentada na Figura $4 \mathrm{~b}$. A avaliação da transferência de calor entre o arranjo de cilindros e a vizinhança é feita através do número de Nusselt para cada cilindro. É possível notar um comportamento bastante semelhante das curvas de $N u_{D}$ em relação aos resultados para os coeficientes de arrasto. Verifica-as que uma maior troca de calor se dá para a maior razão $S_{T} / D$, sendo que este comportamento é esperado, pois os cilindros estando mais afastada a diferença de temperatura entre fluido e os cilindros é evidenciada. Com o espaçamento entre os corpos reduzido, isto é, para as razões menores de $S_{T} / D$, o fluido atinge os cilindros 2 e 3 com uma temperatura mais elevada devido ao ganho de energia pelo contato antecipado com o cilindro 1, diminuindo assim a diferença de temperatura entre os mesmos e o fluido, afetando diretamente no decréscimo do coeficiente de transferência de calor. Outra observação diz respeito ao comportamento quase sobreposto das curvas de $N u_{D}$ para os cilindros 2 e 3. Diferentemente do ocorrido com o coeficiente de arrasto as mesmas são basicamente coincidentes tanto em comportamento quanto em valores. 


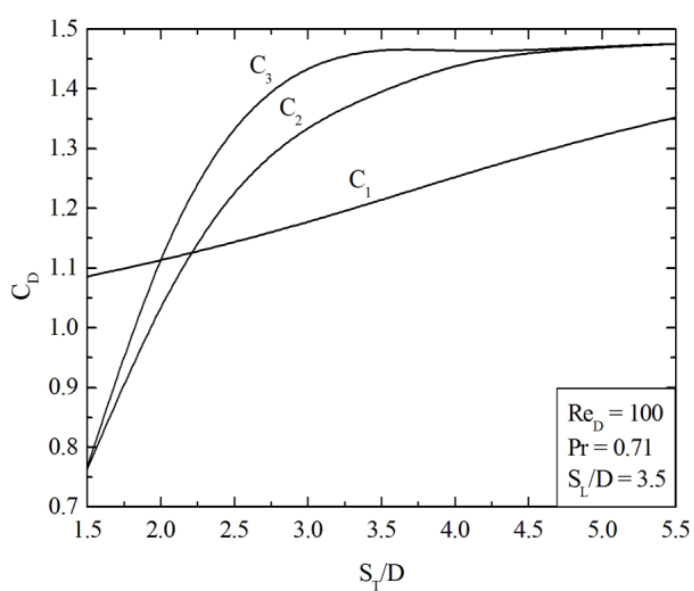

(a)

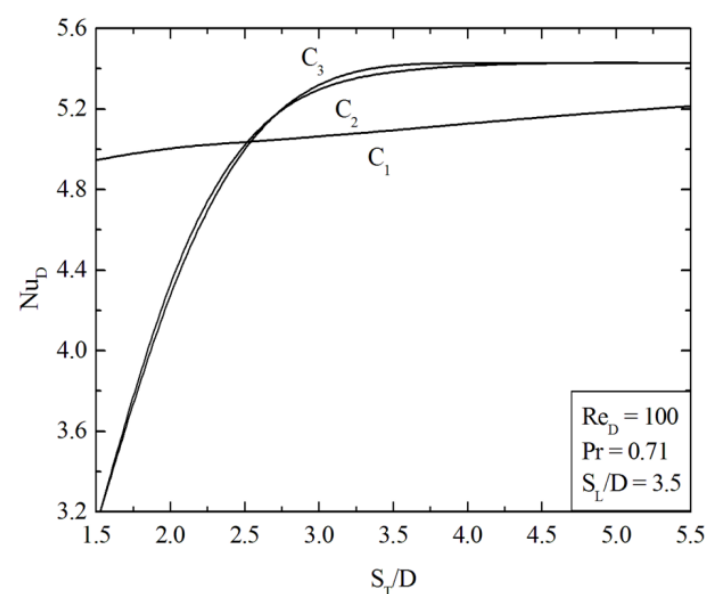

(b)

Figura 4: a) Efeito do parâmetro $S_{T} / D$ sobre o coeficiente de arrasto $\left(C_{D}\right)$ para $S_{L} / D=3,5, \operatorname{Re}_{D}=100 e$ $\operatorname{Pr}=0,71 . b)$ Efeito de $S_{T} / D$ sobre o número de Nusselt $\left(N u_{D}\right)$ para $S_{L} / D=3,5, \operatorname{Re}_{D}=100$ e $\operatorname{Pr}=0,71$

Ao serem realizados os estudos referentes aos outros valores de passo longitudinal $\left(S_{L} / D=\right.$ $1,5 ; 2,0$ e 5,0$)$, em que se realizaram as mesmas variações para o passo transversal $\left(S_{T} / D\right)$, constatou-se que o comportamento térmico e fluidodinâmico manteve-se de acordo com o apresentado para o valor de $S_{L} / D=3,5$. Na Figura 5 pode-se observar claramente a semelhança entre o comportamento para os diferentes casos de $S_{\mathrm{L}} / \mathrm{D}$.

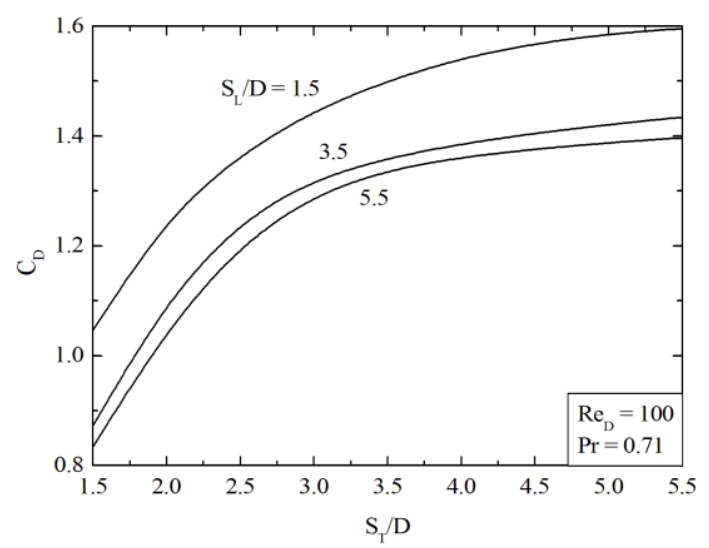

(a)

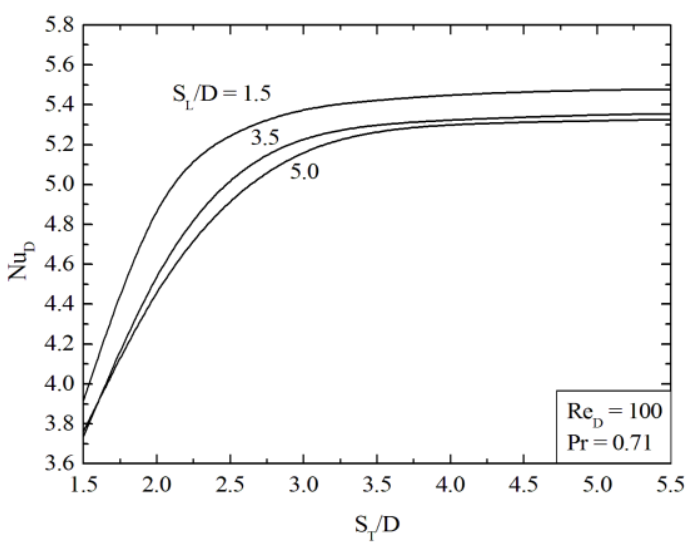

(b)

Figura 5: a) Efeito do parâmetro $S_{T} / D$ sobre o coeficiente de arrasto $\left(C_{D}\right)$ para $S_{L} / D=1,5 ; 3,5$ e 5.0, para um escoamento a $\operatorname{Re}_{D}=100$ e $\mathrm{Pr}=0,71$. b) Efeito do coeficiente de transferência de calor $(\mathrm{Nu})$ para $S_{L} / D=1,5 ; 3,5$ e 5,0, para um escoamento a $\operatorname{Re}_{D}=100$ e $\mathrm{Pr}=0,71$

Comparando as geometrias ótimas para cada razão de $S_{L} / D$, conforme a Figura 6 , verificou-se que a variação deste grau de liberdade não exerce uma influência demasiadamente significativa nos valores de $N u_{D}$ e $C_{D}$. Aliado a isto, os resultados mostraram que a partir de certo momento o aumento da razão de $S_{L} / D$ torna mínima as variações de $N u_{D}$ e $C_{\mathrm{D}}$. Através dos resultados obtidos observa-se que, a geometria que otimiza o $N u_{D}$ é dada para a configuração de $S_{L} / D$ igual a 1,5 e $S_{T} / D$ igual a 5,5 , ao passo que a geometria que otimiza o $C_{D}$ é dada para a configuração de $S_{L} / D$ igual a 5,0 e $S_{T} / D$ igual a 1,5 , mostrando que ao contrario do que pudesse ser esperado, o coeficiente de troca térmica ótimo não foi encontrado para a disposição mais distante entre o arranjo dos cilindros $\left(S_{L} / D=5,0\right.$ e $\left.S_{\mathrm{T}} / \mathrm{D}=5,5\right)$. Em contrapartida, os valores do coeficiente de arrasto se apresentaram de acordo com o previsto. 


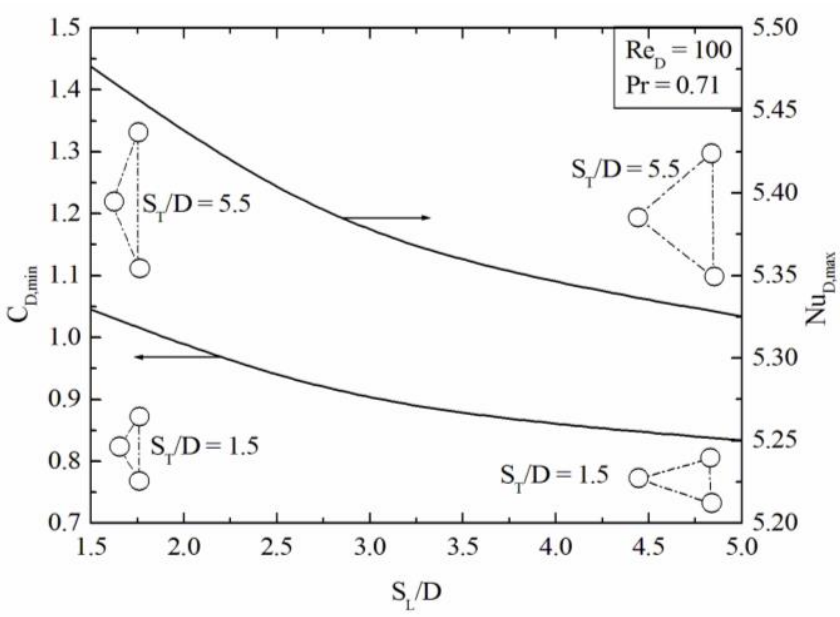

Figura 6: Efeito do parâmetro $S_{L} / D$ sobre o coeficiente de arrasto mínimo $\left(C_{D(M i N)}\right)$ e coeficiente de troca térmica máximo $\left(N u_{D(M A ́ X)}\right)$

Nota-se, a partir dos resultados apresentados na Figura 7, que existe uma região que pode ser considerada ótima, onde mais de uma configuração de $S_{L} / D$ e $S_{T} / D$ ótimas encontram-se nesta área. Para a razão de $S_{L} / D$ igual a 5,0 a curva se aproxima mais da origem, indicando a melhor configuração do arranjo. Vale destacar que através dos resultados obtidos, existe uma tendência de que com o aumento da razão $S_{L} / D$, para uma avaliação multi-objetiva, os resultados tendem a ser mais satisfatórios. No entanto, a partir de um determinado aumento da razão $S_{L} / D$, os efeitos sobre os resultados são desconsideráveis.

Observou-se também, que a geometria ótima multi-objetivo se aproxima da geometria ótima encontrada para coeficiente de arrasto, devido ao fato deste ser mais sensível a variação das razões de $S_{L} / D$ e $S_{T} / D$. Portanto, a geometria ótima para o caso multi-objetivo entre os resultados comparados é encontrada para a razão de $S_{L} / D$ igual a 5,0 e e a variação $2,0 \leq S_{T} / D \leq$ 2,5 , de forma que a configuração $S_{L} / D$ igual a 5,0 e $S_{T} / D$ igual a 2,5 apresenta uma leve vantagem do ponto de vista térmico e $S_{L} / D$ igual a 5,0 e $S_{T} / D$ igual a 2,0 se torna mais benéfica para o problema fluidodinâmico.

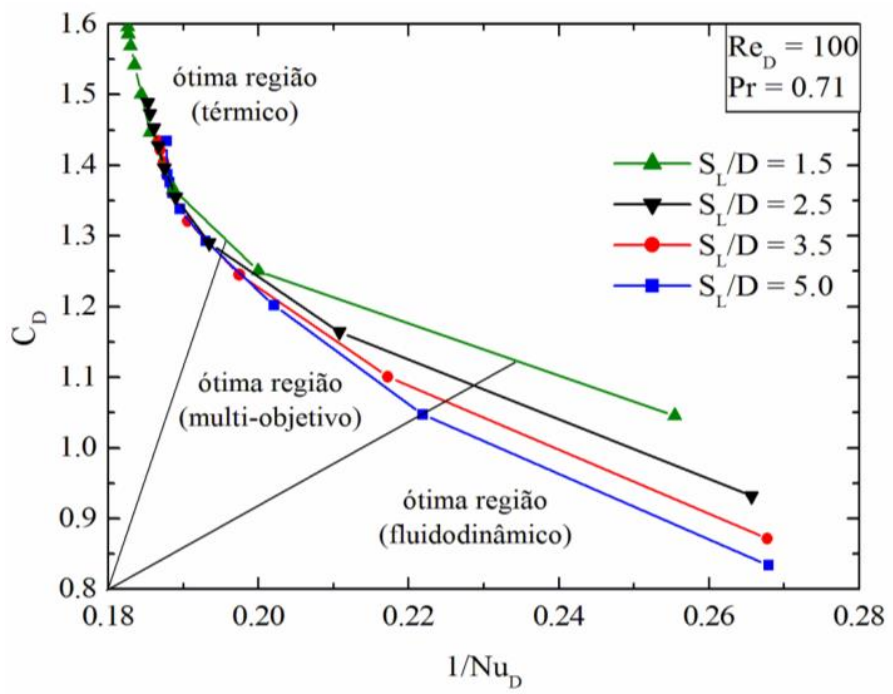

Figura 7: Efeito do parâmetro $S_{T} / D$ sobre o coeficiente de arrasto $\left(C_{D}\right)$ e sobre o inverso do número de Nusselt $\left(\mathrm{Nu}_{D}\right)$ 


\section{CONCLUSÕES}

O presente trabalho avaliou a influência da geometria sobre o comportamento fluidodinâmico e térmico de um arranjo triangular de cilindros sobre o escoamento transiente, bidimensional, incompressível, laminar e com convecção forçada empregando o método Constructal Design. As equações de conservação de massa, quantidade de movimento e energia foram resolvidas com o uso do método de volumes finitos (FVM - do inglês: Finite Volume Method). As simulações foram realizadas para escoamentos com números de Reynolds e Prandtl iguais a 100 e 0,71 , respectivamente. $O$ diâmetro dos três cilindros foi considerado igual em todos os casos. A área ocupada pelos três cilindros foi à restrição geométrica do problema, enquanto as razões $S_{\mathrm{T}} / D$ (passo transversal sobre o diâmetro) e $S_{\mathrm{L}} / D$ (passo longitudinal sobre o diâmetro) foram os graus de liberdade. O principal objetivo do trabalho foi avaliar quais razões $S_{\mathrm{L}} / D$ e $S_{\mathrm{T}} / D$ que minimizam o coeficiente de arrasto e maximizam a taxa de transferência de calor (número de Nusselt), e quais geometrias conduzem a um melhor desempenho para um problema multiobjetivo.

Para os conjuntos de simulações $\left(S_{L} / D=1,5, S_{L} / D=2,5\right.$ e $\left.S_{L} / D=5,0\right)$ ficou comprovado que o comportamento se repete ao comportamento ocorrido para $S_{L} / D$ igual a 3,5 [7] para as mesmas variações das razões $S_{T} / D$. A melhor configuração encontrada para o coeficiente de arrasto foi para as razões $S_{L} / D$ igual a 5,0 e $S_{T} / D$ igual a 1,5 e para o coeficiente de troca térmica o caso otimizado foi $S_{L} / D$ igual a 1,5 e $S_{T} / D$ igual a 5,5 . Para o problema multi-objetivo, uma maior troca térmica com um menor coeficiente de arrasto, encontrou-se uma região ótima, onde se destacou as configurações $S_{L} / D$ igual a 5,0 e $S_{T} / D$ igual a 2,0 e $S_{L} / D=5,0$ e $S_{T} / D$ igual a 2,5 , de forma que a configuração $S_{L} / D$ igual a 5,0 e $S_{T} / D$ igual a 2,5 apresenta uma leve vantagem do ponto de vista térmico e $S_{L} / D$ igual a 5,0 e $S_{T} / D$ igual a 2,0 se torna mais benéfica para o problema fluidodinâmico.

Visto que os casos otimizados obtiveram resultados consideravelmente melhores que as demais configurações, fica evidente a importância desse estudo. Para trabalho futuros é proposto o estudo de outras configurações geométricas e a simulação de escoamentos com outras condições, como a variação do número de Reynolds, e/ou números de Prandtl (simulando outras condições de fluido).

\section{AGRADECIMENTOS}

Os autores L. A. Isoldi, L. A. O. Rocha e E. D. dos Santos agradecem ao CNPq pelo apoio financeiro.

\section{REFERÊNCIAS BIBLIOGRÁFICAS}

1. Bejan A. Convection heat transfer. Durham: John Wiley; 2013. 658 p.

2. Siegel R, Howell JR. Thermal radiation heat transfer. New York: Taylor \& Francis; 2002.

3. Çengel YA, Cimbala JM. Fluid mechanics: fundamentals and application. New York: McGraw-Hill; 2006. 799 p.

4. Sahu AK, Chhabra RP, Eswaran V. Effects of Reynolds and Pradtl numbers on heat transfer from a square cylinder in the unsteady flow regime. Int. J. Heat Mass Transf. 2009 Jan;52(3-4):839-50, doi:10.1016/j.ijheatmasstransfer.2008.07.032

5. Didier E. Numerical simulation of low Reynolds number flows over two circular cylinders in tandem. In: Proceedings of Conference on Modeling Fluid Flow (CMFF); 2009, Budapeste, Hungria.

6. Bello-Ochende T, Bejan A. Constructal multi-scale cylinders with natural convection. Int. J. Heat Mass Transf. 2005 Oct;48(21-22):4300-06, doi:10.1016/j.ijheatmasstransfer.2005.05.023

7. Rocha LAO, Gomes MN, Porte, AF, Galarça MM, Acunha Jr IC, da Silva FMV, Isoldi LA, Dos Santos ED. Constructal Design of turbulent forced convective flows over a pair of circular cylinders. In: Proceedings of Constructal Law Conference (CLC); 2013. Nanjing, China. p. 174-84.

8. Didier E, Parxotomo DM. Análise de uma técnica de redução dos esforços numa estrutura cilíndrica circular. In: Jorge RMN, Tavares JMRS, Alexandre JL, Ferreira AJM, Vaz M, editors. Proceedings of X Congresso Ibero-Americano em Engenharia Mecânica (CIBIM). 2011. Porto, Portugal, p. 659-71. 
9. Bejan A. Shape and structure, from engineering to nature. Cambridge University Press, Cambridge, UK; 2000.

10. Bejan A, Lorente S. Design with Constructal Theory. Hoboken: Wiley; 2008. 527 p.

11. Fluent (version 13.0), ANSYS, Inc.; 2010.

12. Patankar SV. Numerical heat transfer and fluid flow. New York: McGraw-Hill; 1980. 197 p.

13. Versteeg HK, Malalasekera W. An introduction to computational fluid dynamics - the finite volume method. England: Pearson; 2007. 503 p.

14. Hilpert R. Heat transfer from cylinders. Forsch. Geb. Ingenieurwes; 1933. 215 p.

15. Churchill SW, Bernstein MA. Correlation equation for forced convection from gases and liquids to a circular cylinder in cross flow. J. Heat Transfer. 1977 May;99(2):300-6, doi:10.1115/1.3450685

16. Dos Santos ED, Dallagnol A, Petry AP, Rocha LAO. Heat transfer optimization of cross-flow over assemblies of bluff bodies employing constructal principle. In: Proceedings of International Congress of Mechanical Engineering (COBEM); 2009. Gramado, Brazil. 